\title{
Formal Total Synthesis of (-)-Apicularen A via Transannular Conjugate Addition
}

\author{
Ferdow Hilli, Jonathan M. White and Mark Rizzacasa* \\ School of Chemistry, The University of Melbourne, Victoria 3010, Australia \\ masr@unimelb.edu.au
}

Supporting Information 
General. Unless otherwise stated, ${ }^{1} \mathrm{H}$ NMR and proton decoupled ${ }^{13} \mathrm{C}$ NMR spectra were recorded for deuterochloroform solutions with residual chloroform as internal standard. Infrared spectra were run as thin films on $\mathrm{NaCl}$ plates. Optical rotations were recorded for $2 \mathrm{~mL}$ solutions in a $10 \mathrm{~cm}$ microcell. Melting points were determined on a Kofler hot stage apparatus. HRMS (ESI) mass spectra were run at Monash University, Clayton, Victoria.

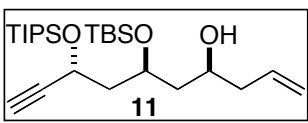

Alkyne 11: colorless oil: [ []$_{\mathrm{D}}-6.4\left(c\right.$ 1.14, $\left.\mathrm{CH}_{2} \mathrm{Cl}_{2}\right)$; IR $v_{\max }$ (film) 3472, 3312, 3078, 2947, 2894, 2868, 2113, 1642, 1257, 1094, 999, $837 \mathrm{~cm}^{-1} ;{ }^{1} \mathrm{H}$ NMR (400 $\left.\mathrm{MHz}, \mathrm{CDCl}_{3}\right) \square 0.132$ (s, 3H), 0.128 (s, 3H), 0.90 (s, 9H), 1.07-1.09 (m, 18H), 1.10-1.18 (m, 3H), $1.56(\mathrm{ddd}, J=14.2,8.8,8.8 \mathrm{~Hz}, 1 \mathrm{H}), 1.77(\mathrm{ddd}, J=14.2,4.0,3.2 \mathrm{~Hz}, 1 \mathrm{H}), 1.92(\mathrm{ddd}, J=13.2,8.4$, $5.8 \mathrm{~Hz}, 1 \mathrm{H}), 2.03$ (ddd, $J=13.2,8.4,4.4 \mathrm{~Hz}, 1 \mathrm{H}), 2.17-2.29(\mathrm{~m}, 2 \mathrm{H}), 2.43(\mathrm{~d}, J=2.0 \mathrm{~Hz}, 1 \mathrm{H}), 2.93$ (s, 1H), 3.85 (br m, 1H), $4.11(\mathrm{~m}, 1 \mathrm{H}), 4.47(\mathrm{ddd}, J=8.4,6.8,2.0,1 \mathrm{H}), 5.10(\mathrm{~d}, J=10.4 \mathrm{~Hz}, 1 \mathrm{H})$, $5.11(\mathrm{~d}, J=16.8 \mathrm{~Hz}, 1 \mathrm{H}), 5.84(\mathrm{dddd}, J=16.8,10.4,7.2,7.2 \mathrm{~Hz}, 1 \mathrm{H}) ;{ }^{13} \mathrm{C} \mathrm{NMR}\left(100 \mathrm{MHz}, \mathrm{CDCl}_{3}\right)$ $\square-4.6,-4.0,12.3,17.9,18.0,18.1,25.8,42.0,42.9,47.1,59.9,69.7,69.9,73.1,85.1,117.5,134.8$; HRMS (ESI) Calcd for $\mathrm{C}_{25} \mathrm{H}_{50} \mathrm{O}_{3} \mathrm{Si}_{2} \mathrm{Na}\left[M+\mathrm{Na}^{+}\right]$: 477.3196, found: 477.3200.

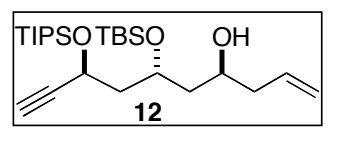

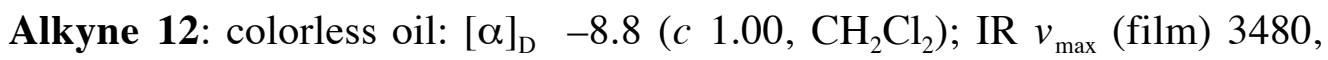
$3304,3054,2947,2894,2868,2306,1642,1266,1060,1000,838 \mathrm{~cm}^{-1} ;{ }^{1} \mathrm{H}$ NMR (400 MHz) $\square 0.11$ (s, 3H), 0.12 (s, 3H), 0.90 (s, 9H), 1.07-1.09 (m, 18H), 1.10-1.18 (m, 3H), 1.64-1.74 (m, 2H), $2.03(\mathrm{ddd}, J=13.2,8.0,5.2 \mathrm{~Hz}, 1 \mathrm{H}), 2.08(\mathrm{ddd}, J=13.2,8.4,6.0 \mathrm{~Hz}, 1 \mathrm{H}), 2.18$ (ddd, $J=14.0,7.2,6.4 \mathrm{~Hz}, 1 \mathrm{H}), 2.27(\mathrm{ddd}, J=14.0,7.2,6.8 \mathrm{~Hz}, 1 \mathrm{H}), 2.43(\mathrm{~d}, J=2.2 \mathrm{~Hz}, 1 \mathrm{H}), 3.43$ (s, 1H), $4.03($ br m, 1H), $4.27(\mathrm{~m}, 1 \mathrm{H}), 4.42(\mathrm{ddd}, J=8.0,6.0,2.2 \mathrm{~Hz}, 1 \mathrm{H}), 5.08(\mathrm{~d}, J=10.0 \mathrm{~Hz}$, 1H), $5.10(\mathrm{dd}, J=17.2,1.6 \mathrm{~Hz}, 1 \mathrm{H}), 5.83$ (dddd, $J=17.2,10.0,7.2,6.8 \mathrm{~Hz}, 1 \mathrm{H}) ;{ }^{13} \mathrm{C} \mathrm{NMR}$ $(100 \mathrm{MHz}) \square-4.8,-4.6,12.3,17.9,18.0,18.1,25.8,40.7,42.3,44.7,60.0,67.9,68.7,73.2,85.0$, 117.4, 134.9; HRMS (ESI) Calcd for $\mathrm{C}_{25} \mathrm{H}_{50} \mathrm{O}_{3} \mathrm{Si}_{2} \mathrm{Na}\left[M+\mathrm{Na}^{+}\right]$: 477.3196, found: 477.3174. 
OTIPSOTBSOH

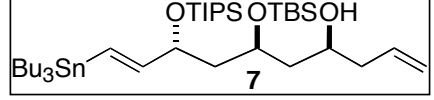

Stannane 7: colorless oil: $[\square]_{\mathrm{D}}-15.6\left(c\right.$ 1.15, $\left.\mathrm{CH}_{2} \mathrm{Cl}_{2}\right)$; IR $v_{\max }$ (film) $3507,3078,2958,2930,2868,1642,1255,1062,994,837 \mathrm{~cm}^{-1} ;{ }^{1} \mathrm{H}$

NMR (400 MHz) $\square 0.09$ (s, 3H), 0.10 (s, 3H), 0.80-0.97 (m, 15H), 0.89 (s, 9H), 1.04-1.05 (m, 21H), 1.30 (sext, $J=7.2 \mathrm{~Hz}, 6 \mathrm{H}), 1.42-1.55(\mathrm{~m}, 6 \mathrm{H}), 1.72-1.86(\mathrm{~m}, 4 \mathrm{H}), 2.21(\mathrm{dd}, J=6.8,6.4 \mathrm{~Hz}, 2 \mathrm{H})$, $3.35(\mathrm{~s}, 1 \mathrm{H}), 3.78-3.84(\mathrm{~m}, 2 \mathrm{H}), 5.08-5.12(\mathrm{~m}, 2 \mathrm{H}), 5.85$ (dddd, $J=16.8,10.4,7.2,6.8 \mathrm{~Hz}, 1 \mathrm{H})$, $5.87(\mathrm{dd}, J=19.2,6.6 \mathrm{~Hz}, 1 \mathrm{H}), 5.97(\mathrm{~d}, J=19.2 \mathrm{~Hz}, 1 \mathrm{H}) ;{ }^{13} \mathrm{C}$ NMR $(100 \mathrm{MHz}) \square-4.6,-3.8,9.4$, $12.3,13.7,17.8,18.0,18.1,25.8,27.3,29.1,41.8,42.5,47.1,70.4,71.1,75.1,117.3,128.9,134.9$, 151.1; HRMS (ESI) Calcd for $\mathrm{C}_{37} \mathrm{H}_{78} \mathrm{O}_{3} \mathrm{Si}_{2} \mathrm{SnNa}\left[M+\mathrm{Na}^{+}\right]$: 767.4409, found: 767.4407.

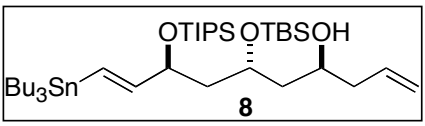

Stannane 8: colorless oil: $[\square]_{\mathrm{D}}+3.7\left(c\right.$ 2.52, $\left.\mathrm{CH}_{2} \mathrm{Cl}_{2}\right)$; IR $v_{\max }$ (film) 3506, 3051, 2956, 2930, 1641, 1265, 1065, 995, $837 \mathrm{~cm}^{-1} ;{ }^{1} \mathrm{H}$ NMR (400 $\mathrm{MHz}) \square 0.07$ (s, 6H), 0.80-0.98 (m, 15H), 0.88 (s, 9H), 1.02-1.07 (m, 21H), 1.29 (sext, J = 7.2 Hz, $6 \mathrm{H}), 1.43-1.56(\mathrm{~m}, 6 \mathrm{H}), 1.61-1.64(\mathrm{~m}, 2 \mathrm{H}), 1.74(\mathrm{ddd}, J=13.6,9.6,4.0 \mathrm{~Hz}, 1 \mathrm{H}), 2.05(\mathrm{ddd}, J=$ 13.6, 10.4, 4.4 Hz, 1H), 2.15 (ddd, $J=13.6,6.8,6.8 \mathrm{~Hz}, 1 \mathrm{H}), 2.28$ (ddd, $J=13.6,7.2,6.8 \mathrm{~Hz}, 1 \mathrm{H}$ ), $3.67(\mathrm{~s}, 1 \mathrm{H}), 3.95-4.00(\mathrm{~m}, 2 \mathrm{H}), 4.04(\mathrm{~m}, 1 \mathrm{H}), 5.09(\mathrm{~d}, J=10.0 \mathrm{~Hz}, 1 \mathrm{H}), 5.10(\mathrm{~d}, J=17.2 \mathrm{~Hz}, 1 \mathrm{H})$, $5.83(\mathrm{dddd}, J=17.2,10.0,7.2,6.8 \mathrm{~Hz}, 1 \mathrm{H}), 5.88(\mathrm{dd}, J=19.2,6.8 \mathrm{~Hz}, 1 \mathrm{H}), 6.00(\mathrm{~d}, J=19.2 \mathrm{~Hz}$, $1 \mathrm{H}) ;{ }^{13} \mathrm{C}$ NMR $(100 \mathrm{MHz}) \square-4.9,-4.5,9.4,12.4,13.7,17.9,18.1,18.14,25.8,27.3,29.1,39.9,42.3$, 44.1, 67.8, 69.3, 75.0, 117.2, 129.1, 135.0, 151.0; HRMS (ESI) Calcd for $\mathrm{C}_{37} \mathrm{H}_{78} \mathrm{O}_{3} \mathrm{Si}_{2} \mathrm{SnNa}$ $\left[M+\mathrm{Na}^{+}\right]:$767.4409, found: 767.4409.

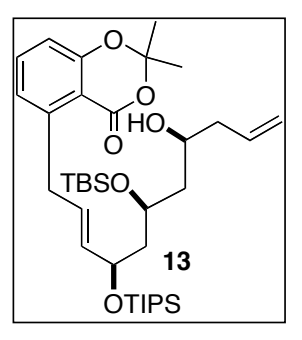

Diene 13: colorless oil: $[\square]_{\mathrm{D}}-17.6\left(c\right.$ 1.07, $\left.\mathrm{CH}_{2} \mathrm{Cl}_{2}\right)$; IR $v_{\max }$ (film) 3504, 3055, 2946, 2866, 1735, 1607, 1266, 1079, 1050, 976, 884, $838 \mathrm{~cm}^{-1} ;{ }^{1} \mathrm{H}$ NMR (400 MHz) $\square 0.07$ (s, 6H), 0.88 (s, 9H), 1.00 (s, 21H), 1.45 (ddd, $J=14.0,9.2,8.8 \mathrm{~Hz}$, 1H), $1.68(\mathrm{ddd}, J=14.0,3.2,2.8 \mathrm{~Hz}, 1 \mathrm{H}), 1.70(\mathrm{~d}, J=4.8 \mathrm{~Hz}, 6 \mathrm{H}), 1.72(\mathrm{ddd}, J=$ 13.2, 8.8, 4.0 Hz, 1H), $1.81(\mathrm{ddd}, J=13.2,9.6,5.2 \mathrm{~Hz}, 1 \mathrm{H}), 2.16(\mathrm{dd}, J=6.8,6.4 \mathrm{~Hz}, 2 \mathrm{H}), 3.20(\mathrm{~s}$, $1 \mathrm{H}), 3.73-3.79(\mathrm{~m}, 2 \mathrm{H}), 3.80-3.89(\mathrm{~m}, 2 \mathrm{H}), 4.11(\mathrm{ddd}, J=13.6,8.4,5.2 \mathrm{~Hz}, 1 \mathrm{H}), 5.05-5.09(\mathrm{~m}, 2 \mathrm{H})$, 
$5.41(\mathrm{dd}, J=15.6,8.0,1 \mathrm{H}), 5.70-5.84(\mathrm{~m}, 2 \mathrm{H}), 6.83(\mathrm{~d}, J=8.0 \mathrm{~Hz}, 1 \mathrm{H}), 6.91(\mathrm{~d}, J=7.6 \mathrm{~Hz}, 1 \mathrm{H})$,

$7.41(\mathrm{t}, J=7.8 \mathrm{~Hz}, 1 \mathrm{H}) ;{ }^{13} \mathrm{C}$ NMR $(100 \mathrm{MHz}) \square-4.6,-3.9,12.4,17.9,18.0,18.1,25.5,25.8,36.7$, $41.8,42.6,47.6,70.1,70.8,71.4,105.1,111.9,115.7,117.3,125.0,129.3,134.7,134.9,135.3$, 145.1, 157.1, 160.1; HRMS (ESI) Calcd for $\mathrm{C}_{36} \mathrm{H}_{62} \mathrm{O}_{6} \mathrm{Si}_{2} \mathrm{Na}\left[M+\mathrm{Na}^{+}\right]$: 669.3983, found: 669.3971 .

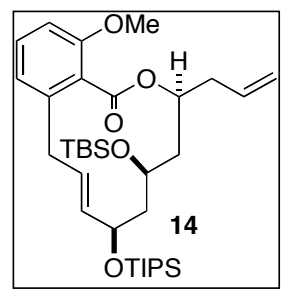

Lactone 14: colorless oil: [ []$_{\mathrm{D}}+47.9\left(c 0.75, \mathrm{CH}_{2} \mathrm{Cl}_{2}\right)$; IR $v_{\max }$ (film) 3054, 2946, 2931, 2895, 2867, 1720, 1644, 1266, 1090, 982, 884, $837 \mathrm{~cm}^{-1} ;{ }^{1} \mathrm{H}$ NMR (400 $\mathrm{MHz}) \square 0.01(\mathrm{~s}, 3 \mathrm{H}), 0.02(\mathrm{~s}, 3 \mathrm{H}), 0.86(\mathrm{~s}, 9 \mathrm{H}), 1.02,(\mathrm{~d}, J=4.0 \mathrm{~Hz}, 21 \mathrm{H}), 1.86$ (dd, $J=14.0,2.8 \mathrm{~Hz}, 1 \mathrm{H}), 1.88(\mathrm{dd}, J=14.0,2.4 \mathrm{~Hz}, 1 \mathrm{H}), 1.99-2.07$ (m, 2H), 2.44-2.56 (m, 2H), 3.19 (app ddd, $J=15.6,2.6,2.0 \mathrm{~Hz}, 1 \mathrm{H}), 3.69$ (dd, $J=15.6,8.8 \mathrm{~Hz}, 1 \mathrm{H}), 3.79$ (s, 3H), 4.10 (m, 1H), 4.53 (br m, 1H), $5.04(\mathrm{~m}, 1 \mathrm{H}), 5.10(\mathrm{dd}, J=10.0,1.6 \mathrm{~Hz}, 1 \mathrm{H}), 5.13(\mathrm{dd}, J=16.8,1.6 \mathrm{~Hz}, 1 \mathrm{H})$, $5.43(\mathrm{dd}, J=15.6,5.2 \mathrm{~Hz}, 1 \mathrm{H}), 5.67(\mathrm{ddd}, J=15.6,8.8,2.6 \mathrm{~Hz}, 1 \mathrm{H}), 5.90(\mathrm{dddd}, J=16.8,10.0$, 7.2, $6.8 \mathrm{~Hz}, 1 \mathrm{H}), 6.79(\mathrm{~d}, J=7.2 \mathrm{~Hz}, 1 \mathrm{H}), 6.80(\mathrm{~d}, J=8.4 \mathrm{~Hz}, 1 \mathrm{H}), 7.25(\mathrm{t}, J=8.0 \mathrm{~Hz}, 1 \mathrm{H}) ;{ }^{13} \mathrm{C}$ NMR (100MHz) $\square 4.5,-4.1,12.3,18.0,18.1,18.2,26.0,36.8,38.1,39.6,43.9,55.6,65.4,69.2$, 72.6, 109.5, 117.2, 122.4, 124.5, 126.4, 130.2, 134.4, 136.3, 138.7, 156.5, 168.3; HRMS (ESI) Calcd for $\mathrm{C}_{34} \mathrm{H}_{58} \mathrm{O}_{5} \mathrm{Si}_{2} \mathrm{Na}\left[M+\mathrm{Na}^{+}\right]$: 625.3720 , found: 625.3730

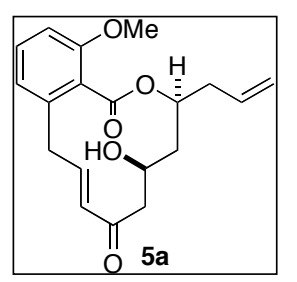

Enone 5a: colorless oil; [ []$_{\mathrm{D}}+76.0\left(c 0.49, \mathrm{CH}_{2} \mathrm{Cl}_{2}\right)$; IR $v_{\max }$ (film) 3453, 3057, $3010,2961,2842,1722,1662,1626,1267,988,897 \mathrm{~cm}^{-1} ;{ }^{1} \mathrm{H}$ NMR $(400 \mathrm{MHz}) \square$ $1.91-2.03(\mathrm{~m}, 2 \mathrm{H}), 2.24(\mathrm{~d}, J=6.0 \mathrm{~Hz}, 1 \mathrm{H}), 2.55(\mathrm{dd}, J=6.8,6.4 \mathrm{~Hz}, 2 \mathrm{H}), 2.71$ $(\mathrm{dd}, J=13.4,7.8 \mathrm{~Hz}, 1 \mathrm{H}), 2.92(\mathrm{dd}, J=13.4,4.8 \mathrm{~Hz}, 1 \mathrm{H}), 3.41(\mathrm{ddd}, J=16.8,4.6,2.0 \mathrm{~Hz}, 1 \mathrm{H})$, $3.82(\mathrm{~s}, 3 \mathrm{H}), 3.93(\mathrm{dd}, J=16.8,8.8 \mathrm{~Hz}, 1 \mathrm{H}), 4.29(\mathrm{~m}, 1 \mathrm{H}), 5.02(\mathrm{~m}, 1 \mathrm{H}), 5.15(\mathrm{~d}, J=10.0 \mathrm{~Hz}, 1 \mathrm{H})$, $5.17(\mathrm{~d}, J=17.2 \mathrm{~Hz}, 1 \mathrm{H}), 5.89$ (dddd, $J=17.2,10.0,7.2,6.8 \mathrm{~Hz}, 1 \mathrm{H}), 6.13(\mathrm{~d}, J=16.0 \mathrm{~Hz}, 1 \mathrm{H})$, $6.76(\mathrm{ddd}, J=16.0,8.8,4.6 \mathrm{~Hz}, 1 \mathrm{H}), 6.82(\mathrm{~d}, J=7.6 \mathrm{~Hz}, 1 \mathrm{H}), 6.87(\mathrm{~d}, J=8.4 \mathrm{~Hz}, 1 \mathrm{H}), 7.31(\mathrm{t}, J=$ 8.0 Hz, 1H); ${ }^{13} \mathrm{C}$ NMR (100MHz, $\square 37.0,38.0,39.4,47.4,55.7,65.8,72.4,110.1,118.1,122.8$, 
123.8, 130.8, 133.1, 133.4, 136.5, 144.5, 157.0, 168.1, 200.5; HRMS (ESI) Calcd for $\mathrm{C}_{19} \mathrm{H}_{22} \mathrm{O}_{5} \mathrm{Na}$ $\left[M+\mathrm{Na}^{+}\right]: 353.1365$, found: 353.1365 .

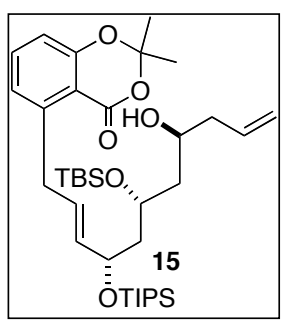

Diene 15: colorless oil: $[\square]_{\mathrm{D}}+12.2\left(c 1.19, \mathrm{CH}_{2} \mathrm{Cl}_{2}\right)$; IR $v_{\max }$ (film) 3477, 3055, $2945,2893,2866,1737,1607,1267,1076,1052,976,884,838 \mathrm{~cm}^{-1} ;{ }^{1} \mathrm{H}$ NMR $(400 \mathrm{MHz}) \square 0.04(\mathrm{~s}, 3 \mathrm{H}), 0.05(\mathrm{~s}, 3 \mathrm{H}), 0.87$ (s, 9H), 1.00 (s, 21H), 1.53-1.63 (m, 2H), $1.70(\mathrm{~d}, J=4.0 \mathrm{~Hz}, 6 \mathrm{H}), 1.71(\mathrm{ddd}, J=13.2,9.2,4.0 \mathrm{~Hz}, 1 \mathrm{H}), 1.99$ (ddd, $J=$ 13.2, 10.0, 4.8 Hz, 1H), $2.13(\mathrm{ddd}, J=13.6,7.2,6.8 \mathrm{~Hz}, 1 \mathrm{H}), 2.25(\mathrm{ddd}, J=13.6,6.8,6.8 \mathrm{~Hz}, 1 \mathrm{H})$, $3.51(\mathrm{~s}, 1 \mathrm{H}), 3.79-3.89(\mathrm{~m}, 2 \mathrm{H}), 3.91(\mathrm{~m}, 1 \mathrm{H}), 4.01($ br m, 1H), 4.06 (ddd, $J=13.6,8.8,5.2 \mathrm{~Hz}$, 1H), 5.05-5.11 (m, 2H), $5.39(\mathrm{dd}, J=15.6,8.4,1 \mathrm{H}), 5.76(\mathrm{ddd}, J=15.6,7.2,6.4,1 \mathrm{H}), 5.81$ (dddd, $J$ $=17.2,10.4,7.2,6.8 \mathrm{~Hz}, 1 \mathrm{H}), 6.83(\mathrm{~d}, J=8.0 \mathrm{~Hz}, 1 \mathrm{H}), 6.91(\mathrm{~d}, J=7.6 \mathrm{~Hz}, 1 \mathrm{H}), 7.41(\mathrm{t}, J=8.0 \mathrm{~Hz}$, $1 \mathrm{H}) ;{ }^{13} \mathrm{C}$ NMR $(100 \mathrm{MHz}) \square-4.9,-4.6,12.4,17.9,18.06,18.1,25.5,25.8,36.7,40.4,42.3,44.9$, 67.8, 68.9, 71.4, 105.1, 111.9, 115.7, 117.2, 125.1, 129.4, 134.6, 135.0, 135.3, 145.0, 157.1, 160.1; HRMS (ESI) Calcd for $\mathrm{C}_{36} \mathrm{H}_{62} \mathrm{O}_{6} \mathrm{Si}_{2} \mathrm{Na}\left[M+\mathrm{Na}^{+}\right]$: 669.3983, found: 669.3969 .

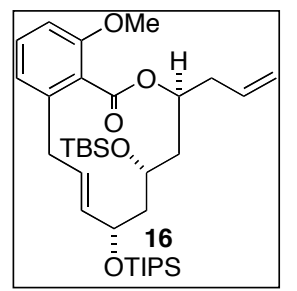

Lactone 16: colorless oil: $[\square]_{\mathrm{D}}+21.9\left(c 1.90, \mathrm{CH}_{2} \mathrm{Cl}_{2}\right)$; IR $v_{\max }$ (film) 3076,3053 , 2946, 2894, 2866, 1725, 1644, 1277, 1085, 975, 911, $837 \mathrm{~cm}^{-1} ;{ }^{1} \mathrm{H}$ NMR (400 $\mathrm{MHz}) \square 0.14(\mathrm{~s}, 3 \mathrm{H}), 0.22(\mathrm{~s}, 3 \mathrm{H}), 0.91(\mathrm{~s}, 9 \mathrm{H}), 1.03(\mathrm{~s}, 21 \mathrm{H}), 1.58$ (dd, $J=15.2$, $6.8 \mathrm{~Hz}, 1 \mathrm{H}), 1.70(\mathrm{ddd}, J=12.8,10.0,3.6 \mathrm{~Hz}, 1 \mathrm{H}), 1.73(\mathrm{dd}, J=15.2,8.0 \mathrm{~Hz}, 1 \mathrm{H}), 1.84(\mathrm{ddd}, J=$ 12.8, 11.6, $5.2 \mathrm{~Hz}, 1 \mathrm{H}), 2.30(\mathrm{~m}, 1 \mathrm{H}), 2.46(\mathrm{~m}, 1 \mathrm{H}), 3.37(\mathrm{~d}, J=16.4 \mathrm{~Hz}, 1 \mathrm{H}), 3.74(\mathrm{ddd}, J=16.4$, 5.6, $2.4 \mathrm{~Hz}, 1 \mathrm{H}), 3.80(\mathrm{~s}, 3 \mathrm{H}), 4.03-4.11(\mathrm{~m}, 2 \mathrm{H}), 5.09(\mathrm{dd}, J=10.0,1.6 \mathrm{~Hz}, 1 \mathrm{H}), 5.13(\mathrm{dd}, J=$ 17.2, 1.6 Hz, 1H), $5.28(\operatorname{app~q}, J=7.2 \mathrm{~Hz}, 1 \mathrm{H}), 5.42-5.50(\mathrm{~m}, 2 \mathrm{H}), 5.88$ (dddd, $J=17.2,10.0,6.4$, $6.0 \mathrm{~Hz}, 1 \mathrm{H}), 6.79(\mathrm{~d}, J=7.6 \mathrm{~Hz}, 1 \mathrm{H}), 6.81(\mathrm{~d}, J=8.4 \mathrm{~Hz}, 1 \mathrm{H}), 7.24(\mathrm{t}, J=8.0 \mathrm{~Hz}, 1 \mathrm{H}) ;{ }^{13} \mathrm{C} \mathrm{NMR}$ $(100 \mathrm{MHz}) \square-4.5,-4.1,12.3,17.9,18.0,18.1,25.9,36.8,40.0,42.5,44.4,55.5,68.8,72.1,74.5$, 109.4, 117.2, 122.7, 124.5, 129.6, 130.0, 134.2, 135.2, 138.5, 156.6, 167.9; HRMS (ESI) Calcd for $\mathrm{C}_{34} \mathrm{H}_{58} \mathrm{O}_{5} \mathrm{Si}_{2} \mathrm{Na}\left[M+\mathrm{Na}^{+}\right]: 625.3720$, found: 625.3716 . 


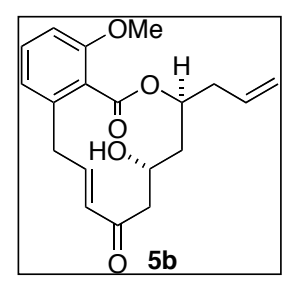

Enone 5b: colorless oil: [ []$_{\mathrm{D}}-18.4\left(c 0.91, \mathrm{CH}_{2} \mathrm{Cl}_{2}\right)$; IR $v_{\max }$ (film) 3460, 3056, 2986, 2964, 2931, 2842, 1720, 1660, 1633, 1266, 984, $896 \mathrm{~cm}^{-1} ;{ }^{1} \mathrm{H}$ NMR (400 $\mathrm{MHz}) \square 1.67(\mathrm{ddd}, J=16.0,6.8,6.4 \mathrm{~Hz}, 1 \mathrm{H}), 1.93(\mathrm{ddd}, J=16.0,5.6,2.4 \mathrm{~Hz}$, $1 \mathrm{H}), 2.40-2.50(\mathrm{~m}, 2 \mathrm{H}), 2.65(\mathrm{dd}, J=13.2,8.6 \mathrm{~Hz}, 1 \mathrm{H}), 2.97(\mathrm{dd}, J=13.2,4.0 \mathrm{~Hz}, 1 \mathrm{H}), 3.43$ (ddd, $J=16.0,4.0,1.8 \mathrm{~Hz}, 1 \mathrm{H}), 3.80(\mathrm{~s}, 3 \mathrm{H}), 3.85(\mathrm{dd}, J=16.0,10.0 \mathrm{~Hz}, 1 \mathrm{H}), 4.37(\mathrm{~m}, 1 \mathrm{H}), 5.11-5.19$ (m, 3H), 5.85 (dddd, $J=17.2,10.0,6.8,6.8 \mathrm{~Hz}, 1 \mathrm{H}), 6.23(\mathrm{dd}, J=16.0,1.8 \mathrm{~Hz}, 1 \mathrm{H}), 6.68(\mathrm{ddd}, J=$ 16.0, 10.0, 4.0 Hz, 1H), 6.81-6.86 (m, 2H), $7.30(\mathrm{t}, J=8.0 \mathrm{~Hz}, 1 \mathrm{H}) ;{ }^{13} \mathrm{C}$ NMR $(100 \mathrm{MHz}) \square 73.0$, $39.8,41.7,48.0,55.7,69.1,73.6,110.1,118.4,122.9,124.1,130.7,133.0,133.9,136.6,143.4$, 157.0, 168.2, 200.6; HRMS (ESI) Calcd for $\mathrm{C}_{19} \mathrm{H}_{22} \mathrm{O}_{5} \mathrm{Na}\left[M+\mathrm{Na}^{+}\right]$: 353.1365, found: 353.1372 .

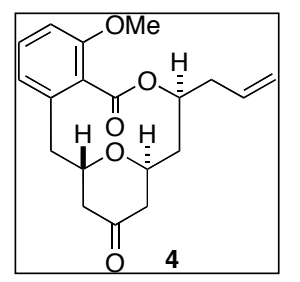

Pyranone 4: colorless crystalline solid: m.p. $182-183^{\circ} \mathrm{C}$ (EtOAc/petrol); $[\square]_{\mathrm{D}}$ $+138.0\left(c 0.53, \mathrm{CHCl}_{3}\right)$, lit. $^{1}$ (enantiomer) $[\square]_{\mathrm{D}}-140\left(c 0.60, \mathrm{CHCl}_{3}\right) ; \mathrm{IR} v_{\max }($ film) $3057,2960,2927,2854,1719,1644,1599,1471,1266,1117,1074 \mathrm{~cm}^{-1} ;{ }^{1} \mathrm{H}$ NMR $(400 \mathrm{MHz}) \square 1.72(\mathrm{ddd}, J=14.8,3.2,1.2 \mathrm{~Hz}, 1 \mathrm{H}), 1.83(\mathrm{ddd}, J=14.8,11.2,10.0 \mathrm{~Hz}, 1 \mathrm{H}), 2.22$ (dd, $J=17.6,10.8 \mathrm{~Hz}, 1 \mathrm{H}), 2.37(\mathrm{~m}, 1 \mathrm{H}), 2.43(\mathrm{dd}, J=17.6,3.6 \mathrm{~Hz}, 1 \mathrm{H}), 2.48(\mathrm{~m}, 1 \mathrm{H}), 2.483$ (dd, $J=14.0,2.0 \mathrm{~Hz}, 1 \mathrm{H}), 2.55(\mathrm{dd}, J=15.2,8.8 \mathrm{~Hz}, 1 \mathrm{H}), 2.66(\mathrm{dd}, J=15.2,6.4 \mathrm{~Hz}, 1 \mathrm{H}), 3.46(\mathrm{dd}, J=$ 14.0, 11.6 Hz, 1H), 4.25 (dddd, $J=15.2,8.8,6.4,2.0 \mathrm{~Hz}, 1 \mathrm{H}), 4.50(\mathrm{app} \mathrm{dt}, J=10.0,3.6 \mathrm{~Hz}, 1 \mathrm{H})$, 5.15, (dd, $J=10.0,1.6 \mathrm{~Hz}, 1 \mathrm{H}), 5.18(\mathrm{dd}, J=16.8,1.6 \mathrm{~Hz}, 1 \mathrm{H}), 5.63(\mathrm{ddd}, J=11.2,8.4,5.2,3.2$ Hz, 1H), 5.88 (dddd, $J=16.8,10.0,7.2,6.8 \mathrm{~Hz}, 1 \mathrm{H}), 6.79$ (d, $J=7.6 \mathrm{~Hz}, 1 \mathrm{H}), 6.84(\mathrm{~d}, J=8.4 \mathrm{~Hz}$, 1H), $7.28(\mathrm{dd}, J=8.4,7.6 \mathrm{~Hz}, 1 \mathrm{H}) ;{ }^{13} \mathrm{C} \mathrm{NMR}(100 \mathrm{MHz}) \square 38.9,39.0,40.1,47.1,55.8,68.1,72.1$, 74.0, 110.0, 118.0, 122.6, 125.6, 130.5, 133.3, 147.0, 155.9, 169.8, 207.1; HRMS (ESI) Calcd for $\mathrm{C}_{19} \mathrm{H}_{22} \mathrm{O}_{5} \mathrm{Na}\left[M+\mathrm{Na}^{+}\right]:$353.1365, found: 353.1372 .

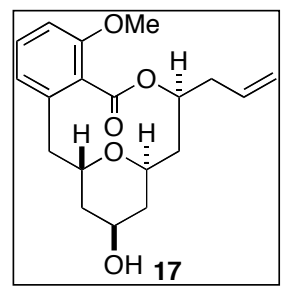

Alcohol 17: colorless oil: $[\square]_{\mathrm{D}}+18.1\left(\right.$ c $\left.0.63, \mathrm{CHCl}_{3}\right)$; IR $v_{\max }$ (film) 3427, 3056, 2978, 2924, 2857, 1715, 1470, 1266, 1114, 1096, 1073, 996, $897 \mathrm{~cm}^{-1} ;{ }^{1} \mathrm{H}$ NMR $(400 \mathrm{MHz}) \square 1.36(\mathrm{ddd}, J=12.4,8.0,7.6 \mathrm{~Hz}, 1 \mathrm{H}), 1.59$ (d, $J=6.0 \mathrm{~Hz}, 1 \mathrm{H}), 7.71$ 
$(\mathrm{dd}, J=14.4,4.0 \mathrm{~Hz}, 1 \mathrm{H}), 1.78-1.92(\mathrm{~m}, 4 \mathrm{H}), 2.34(\mathrm{dd}, J=14.0,1.8 \mathrm{~Hz}, 1 \mathrm{H}), 2.35$ (m, 1H), 2.47

(ddd, $J=14.8,8.0,6.8 \mathrm{~Hz}, 1 \mathrm{H}), 3.37(\mathrm{dd}, J=14.0,12.0 \mathrm{~Hz}, 1 \mathrm{H}), 3.79(\mathrm{~s}, 3 \mathrm{H}), 3.94-4.02(\mathrm{~m}, 2 \mathrm{H})$, $4.17(\mathrm{~m}, 1 \mathrm{H}), 5.12-5.19(\mathrm{~m}, 2 \mathrm{H}), 5.60(\mathrm{~m}, 1 \mathrm{H}), 5.87(\mathrm{dddd}, J=17.2,10.0,6.8,6.8 \mathrm{~Hz}, 1 \mathrm{H}), 6.80(\mathrm{~d}$, $J=7.2 \mathrm{~Hz}, 1 \mathrm{H}), 6.81(\mathrm{~d}, J=9.2 \mathrm{~Hz}, 1 \mathrm{H}), 7.26(\mathrm{dd}, J=9.2,7.2 \mathrm{~Hz}, 1 \mathrm{H}) ;{ }^{13} \mathrm{C} \mathrm{NMR}(100 \mathrm{MHz}) \square$ 36.2, 37.7, 38.9, 40.6, 41.0 55.8, 65.4, 66.9, 73.1, 74.8, 109.5, 117.7, 122.7, 125.7, 130.2, 133.5, 138.6, 155.6, 170.3; HRMS (ESI) Calcd for $\mathrm{C}_{19} \mathrm{H}_{24} \mathrm{O}_{5} \mathrm{Na}\left[M+\mathrm{Na}^{+}\right]$: 355.1521, found: 355.1517 .

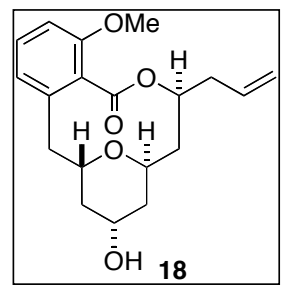

Alcohol 18: colorless oil: $[\square]_{\mathrm{D}}+10.7\left(c \quad 0.49, \mathrm{CHCl}_{3}\right)$, lit. ${ }^{1}$ (enantiomer) $[\square]_{\mathrm{D}}$ -10.6 (c 1.50, $\mathrm{CHCl}_{3}$ ); IR $v_{\max }$ (film) 3427, 3056, 2942, 2924, 2841, 1716, 1470, 1266, 1115, 1095, 1072, 996, $896 \mathrm{~cm}^{-1} ;{ }^{1} \mathrm{H}$ NMR (400 MHz) $\square 1.51-1.59$ (m, 3H), $1.78(\mathrm{ddd}, J=12.8,6.6,6.0 \mathrm{~Hz}, 1 \mathrm{H}), 1.91(\mathrm{ddd}, J=14.8,11.4,10.8 \mathrm{~Hz}, 1 \mathrm{H}), 1.99(\mathrm{ddd}, J=12.8$, 4.4, $4.4 \mathrm{~Hz}, 1 \mathrm{H}), 2.34(\mathrm{ddd}, J=14.4,6.8,5.6 \mathrm{~Hz}, 1 \mathrm{H}), 2.45(\mathrm{~m}, 1 \mathrm{H}), 2.46(\mathrm{~d}, J=14.8 \mathrm{~Hz}, 1 \mathrm{H}), 3.39$ $(\mathrm{dd}, J=14.8,10.0 \mathrm{~Hz}, 1 \mathrm{H}), 3.97-4.08(\mathrm{~m}, 2 \mathrm{H}), 4.32(\mathrm{~m}, 1 \mathrm{H}), 5.10-5.18(\mathrm{~m}, 2 \mathrm{H}), 5.59(\mathrm{dddd}, J=$ 13.2, 10.8, 5.6, 5.6, $2.4 \mathrm{~Hz}, 1 \mathrm{H}), 5.87$ (dddd, $J=17.2,10.4,7.2,6.8 \mathrm{~Hz}, 1 \mathrm{H}), 6.73(\mathrm{~d}, J=7.6 \mathrm{~Hz}$, $1 \mathrm{H}), 6.78(\mathrm{~d}, J=8.0 \mathrm{~Hz}, 1 \mathrm{H}), 7.22(\mathrm{t}, J=8.0 \mathrm{~Hz}, 1 \mathrm{H}) ;{ }^{13} \mathrm{C} \mathrm{NMR}(100 \mathrm{MHz}) \square 38.1,38.7,38.9,39.4$, $39.6,55.8,65.1,67.5,72.6,72.8,109.4,117.7,122.8,125.4,129.9,133.6,138.4,155.9,169.1$; HRMS (ESI) Calcd for $\mathrm{C}_{19} \mathrm{H}_{24} \mathrm{O}_{5} \mathrm{Na}\left[M+\mathrm{Na}^{+}\right]$: 355.1521, found: 355.1517 .

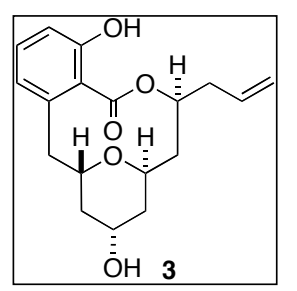

Diol 3: pale yellow gum: $[\square]_{\mathrm{D}}+5.6(c 0.41, \mathrm{MeOH})$, lit. $^{2}[\square]_{\mathrm{D}}+6.8(c 0.16$, $\mathrm{MeOH})$, lit. $^{1}$ (enantiomer) $[\square]_{\mathrm{D}}-4.5\left(c\right.$ 0.15, MeOH); ${ }^{1} \mathrm{H}$ NMR (400 MHz, $d_{\sigma^{-}}$ acetone) $\square 1.48(\mathrm{ddd}, J=12.8,8.4,8.4 \mathrm{~Hz}, 1 \mathrm{H}), 1.50(\mathrm{ddd}, J=12.8,8.0,4.8 \mathrm{~Hz}$, 1H), 1.57 (ddd, $J=14.8,2.4,2.0 \mathrm{~Hz}, 1 \mathrm{H}), 1.67(\mathrm{ddd}, J=12.8,7.2,6.8 \mathrm{~Hz}, 1 \mathrm{H}), 1.83$ (ddd, $J=14.8$, $10.8,10.6 \mathrm{~Hz}, 1 \mathrm{H}), 1.92(\mathrm{ddd}, J=12.8,4.4,4.4 \mathrm{~Hz}, 1 \mathrm{H}), 2.29-2.41(\mathrm{~m}, 2 \mathrm{H}), 2.43(\mathrm{dd}, J=14.8,1.2$ $\mathrm{Hz}, 1 \mathrm{H}), 3.33(\mathrm{dd}, J=14.8,9.6 \mathrm{~Hz}, 1 \mathrm{H}), 3.79$ (d, $J=4.4 \mathrm{~Hz}, 1 \mathrm{H}), 3.87(\mathrm{~m}, 1 \mathrm{H}), 3.98(\mathrm{~m}, 1 \mathrm{H}), 4.26$ (m, 1H), 5.03 (dddd, $J=10.2,2.0,1.6,1.6 \mathrm{~Hz}, 1 \mathrm{H}), 5.13(\mathrm{dddd}, J=17.2,2.0,1.6,1.6 \mathrm{~Hz}, 1 \mathrm{H}), 5.47$ (dddd, $J=10.6,5.6,5.6,2.4 \mathrm{~Hz}, 1 \mathrm{H}), 5.91(\mathrm{dddd}, J=17.2,10.2,7.2,6.8 \mathrm{~Hz}, 1 \mathrm{H}), 6.68(\mathrm{~d}, J=7.6$ 
$\mathrm{Hz}, 1 \mathrm{H}), 6.76(\mathrm{~d}, J=8.0 \mathrm{~Hz}, 1 \mathrm{H}), 7.10(\mathrm{dd}, J=8.0,7.6 \mathrm{~Hz}, 1 \mathrm{H}), 8.39(\mathrm{~s}, 1 \mathrm{H}) ;{ }^{13} \mathrm{C} \mathrm{NMR}(100 \mathrm{MHz}$, $d_{6}$-acetone) $\square 39.0,39.7,39.9,40.1,40.3,64.8,68.0,73.6,73.5,114.3,117.4,122.3,125.4,130.2$,

135.3, 140.2, 154.3, 169.2; HRMS (ESI) Calcd for $\mathrm{C}_{18} \mathrm{H}_{22} \mathrm{O}_{5} \mathrm{Na}\left[M+\mathrm{Na}^{+}\right]$: 341.1365 , found: 341.1364.

\section{References}

(1) Lewis, A.; Stefanuti, I.; Swain, S. A.; Smith, S. A.; Taylor, R. J. K. Org. Biomol. Chem. 2003, $1,104$.

(2) Bhattacharjee, A.; De Brabander, J. K. Tetrahedron Lett. 2000, 41, 8069. 